\title{
Residual Dizziness after Benign Paroxysmal Positional Vertigo
}

\author{
Munyoung Chang (iD) and Seog-Kyun Mun (iD \\ Department of Otorhinolaryngology-Head and Neck Surgery, College of Medicine, Chung-Ang University, Seoul, Korea
}

\section{양성발작성두위현훈 후 잔존 어지럼}

\author{
장문영 · 문석균 \\ 중앙대학교 의과대학 이비인후과학교실
}

\author{
Received August 30, 2020 \\ Accepted September 7, 2020 \\ Address for correspondence \\ Seog-Kyun Mun, MD, PhD \\ Department of Otorhinolaryngology- \\ Head and Neck Surgery, \\ College of Medicine, \\ Chung-Ang University, \\ 102 Heukseok-ro, Dongjak-gu, \\ Seoul 06973, Korea \\ Tel +82-2-6299-1765 \\ Fax +82-2-825-1765 \\ E-mail entdoctor@cau.ac.kr
}

Benign paroxysmal positional vertigo (BPPV) is one of the most common diseases that cause dizziness. It can be resolved relatively easily using canalith-repositioning maneuvers. However, some patients suffer from dizziness even after successful canalith-repositioning. This is an atypical dizziness felt in the absence of typical nystagmus after resolution of BPPV and named residual dizziness. Otolithic organ disorder, anxiety and persistence of debris in the canal have been reported to be associated with residual dizziness. Although 31-61\% of BPPV patients suffer from residual dizziness, residual dizziness has not yet been clearly known not only the treatment but also the pathophysiology. This article investigate the factors associated with residual dizziness and treatment and prediction methods for residual dizziness.

Korean J Otorhinolaryngol-Head Neck Surg 2020;63(10):443-7

Key Words Anxiety · Benign paroxysmal positional vertigo - Dizziness ·

Otolithic membrane.

\section{서 론}

양성발작성두위현훈(benign paroxysmal positional vertigo, BPPV)은 어지럼을 유발하는 가장 흔한 질환 중 하나 로, ${ }^{1)}$ 이석기관에서 반고리관으로 떨어져 나온 이석에 의해 발생한다. ${ }^{2)} \mathrm{BPPV}$ 는 이석정복술을 통해 비교적 쉽게 치료할 수 있다. ${ }^{3)}$ 그러나 명확한 발생 기전과 치료 방법에도 불구하 고, 일부 환자들에서는 성공적인 이석정복술 후에도 비전형 적인 어지럼이 지속된다. 이는 $\mathrm{BPPV}$ 가 완치된 후 $\mathrm{BPPV}$ 의 전형적인 어지럼이나 안진이 없는 상태에서 느껴지는 비전형 적인 어지럼으로 잔존 어지럼(residual dizziness)이라고 불 린다." 잔존 어지럼은 BPPV 환자의 31 61\%에서 발생하며,-7) 며칠에서 몇 주까지 지속되는 것으로 알려져 있다. ${ }^{8)}$

This is an Open Access article distributed under the terms of the Creative Commons Attribution Non-Commercial License (https://creativecommons.org/licenses/by-nc/4.0) which permits unrestricted non-commercial use, distribution, and reproduction in any medium, provided the original work is properly cited.

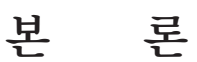

잔존 어지럼은 비교적 최근에 알려진 질환으로, 최근 들어 활발한 연구가 진행되고 있다. 본 논문에서는 잔존 어지럼의 관련 인자, 예측, 약물 치료의 효과에 대해 살펴보았다.

\section{잔존 어지럼의 관련 인자}

잔존 어지럼과 관련된 인자 및 원인을 찾기 위해 여러 연 구가 수행되었다. 그 결과, $\mathrm{BPPV}$ 의 지속 기간,6,99) 고령, ${ }^{10-12)}$ 불안감(anxiety), ${ }^{9,12-14)}$ 이석 기관 장애(otolithic organ disor$\mathrm{der})^{15-17)}$ 등이 잔존 어지럼과 연관되어 있는 것으로 보고되었 다. 반면 BPPV가 발생한 반고리관, 성별, 이석정복술의 시행 횟수, 안진의 세기, 고혈압, 당뇨병, 심장 질환, 고지혈증 등의 기저 질환은 잔존 어지럼과 관계없는 것으로 보고되었다. ${ }^{47}$ 여기서는 잔존 어지럼의 원인으로 제안되고 있는 이석 기관 
장애, 불안감 등에 대해 좀더 알아보고자 한다.

\section{이석 기관 장애(otolithic organ disorder)}

잔존 어지럼과 연관이 있는 것으로 보고된 여러 인자들 중, 이석 기관 장애에 대한 연구가 많이 이루어져 있다. 여러 연구 에서 이석 기관 장애와 잔존 어지럼 사이의 연관 관계를 보 고하였다. BPPV는 이석 기관에서 떨어져 나온 이석에 의해 발생하므로, BPPV 환자는 이석 기관 장애를 동반하고 있을 가능성이 있다. 따라서 이석정복술로 BPPV가 치료된 뒤에도 이석 기관 장애는 지속될 수 있고, 이는 잔존 어지럼으로 이 어질 가능성이 있다. ${ }^{8,18-21)}$

이석 기관 장애는 여러 가지 검사로 진단이 가능하며, 대표 적인 검사 방법으로 subjective visual vertical(SVV) 검사가 있다.22) 먼저 $\mathrm{BPPV}$ 환자에서 SVV 검사를 시행한 결과를 살 펴보면, Böhmer와 Rickenmann ${ }^{23)}$ 은 치료받지 않은 BPPV 환자 19 명에게 SVV 검사를 시행하였고, 1 명의 환자만이 비 정상 소견을 보였다. von Brevern 등 ${ }^{16}$ 도 12 명의 BPPV 환자 와 24명의 정상인에게 SVV 검사를 시행하였는데, 두 그룹 간에 차이가 없었다. 또한 Faralli 등은 BPPV 환자에서 SVV 이상 소견이 발견되나, 짧은 기간 내에 회복되기 때문에 이는 잔존 어지럼의 원인이 아닐 것으로 생각하였다. 반면, Gall 등 $^{24)}$ 은 $\mathrm{BPPV}$ 환자 16 명 중 14 명에서 SVV 검사상 이상 소견 이 있음을 보고하여, 상반된 결과를 보였다. 위의 4가지 연구 모두 포함된 대상자 수가 많지 않아 BPPV 환자에서의 SVV 결과에 대해서는 추가 연구가 필요하다.

이석 기관 장애를 평가할 수 있는 또 다른 검사 방법으로 vestibular evoked myogenic potential(VEMP) 검사가 있다. VEMP를 유발하는 신경 경로에 구형낭 혹은 난형낭이 포함 되어 있어 VEMP 검사를 통해 이석 기관 장애를 추정할 수 있다. ${ }^{25)}$ Yetiser 등 ${ }^{17)}$ 은 어지럼의 지속 기간이 짧았던 BPPV 환자에 비해 어지럼이 오랜 기간 지속된 BPPV 환자에서 cervical VEMP(cVEMP)의 p1 latency가 길었다고 보고했고, $\mathrm{cVEMP}$ 의 결과가 잔존 어지럼과 연관되어 있다고 제안했다. $\mathrm{SeO}$ 등 $^{15)}$ 은 잔존 어지럼이 ocular VEMP 결과와 연관되어 있으며 지속되는 난형낭 기능장애(utricular dysfunction)로 인해 발생한다고 주장하였다.

이외에도 von Brevern 등히는 정상인과 $\mathrm{BPPV}$ 환자에서 otolith-ocular reflex를 측정하였고, 정상인에 비해 BPPV 환자에서 otolith-ocular reflex가 감소해 있고 BPPV 회복 후 한 달 후에도 otolith-ocular reflex 감소가 지속됐다고 보고했다. 이 결과를 바탕으로 난형낭 기능 장애가 BPPV 발 생과 연관되어 있고, 이것이 잔존 어지럼의 원인이라고 제안 했다.
이렇듯 여러 연구에서 이석 기관 장애가 잔존 어지럼의 원 인일 것으로 추정하였으며 여러 근거가 제시되어 있다. 이석 기관 장애라는 하나의 병인으로 잔존 어지럼이 발생한다고 단정지을 수는 없으나, 이석 기관 장애가 잔존 어지럼 발생에 중요한 역할을 할 것으로 추정된다.

\section{불안감(anxiety)}

Teggi 등른 근 72 세 이상의 고령, BPPV 증상이 9일 이상 지 속된 경우, 불안감이 큰 경우에 잔존 어지럼 발생이 높은 것 으로 보고하였다. Faralli 등 ${ }^{26}$ 은 불안감이 잔존 어지럼 발생 과 연관되어 있고, 불안감은 $\mathrm{BPPV}$ 의 지속 기간, 나이, 성별과 연관되어 있음을 보고하였다. Jung 등흐는 항불안제가 잔존 어지럼 발생을 줄일 수 있다고 보고하였다. 여러 연구에서 불 안감과 잔존 어지럼 간의 연과성을 보고하여, 불안감이 잔존 어지럼 발생에 영향을 미칠 것으로 생각된다.

\section{기타 요인}

이 외의 요인들도 잔존 어지럼의 원인으로 제시되었다. Faralli 등은 중추 신경계가 BPPV가 있는 상태에 적응하였 다가 BPPV가 완치된 상태에 적응하지 못해 잔존 어지럼이 발생한다고 제안하였다. 즉, $\mathrm{BPPV}$ 가 있는 상태에서는 반고 리관 안에 이석이 떠다니고 있어 이석이 있는 반고리관에서 기인하는 전기적 발화가 높아져 전정핵(vestibular nucleus)의 활성도가 올라가게 되고, 뇌가 이를 낮추는 방향으로 적응을 하게 된다. 그런데 이석정복술로 BPPV가 치료되고 나면 이 석에 의한 전기적 발화가 줄어들게 되는데, 뇌가 변화된 상 황에 빠르게 적응하지 못하여 잔존 어지럼이 발생한다는 것 이다. Inagaki 등 ${ }^{27)}$ 은 떨어져 나온 이석이 이석정복술로 다시 난형낭으로 돌아감으로써 잔존 어지럼이 발생한다고 제안하 였다. Prokopakis 등이이 이를 구체화하였는데, 이석이 난형 낭에서 떨어져 나왔다가 다시 붙을 때 난형낭에 가해지는 압 력에 차이가 생기고, 이는 난형낭의 감각 세포 자극의 변화로 이어져 잔존 어지럼이 발생한다는 것이다. Di Girolamo 등 29 은 이석정복술 후에도 소량의 이석이 반고리관 내에 남아 잔 존 어지럼을 유발한다고 제안하였다. Kim과 Lee근 잔존 어 지럼을 호소하는 환자에서 기립성 저혈압이 더 많이 발견된 다는 것을 보고하였다. 이를 바탕으로, 자세 변화 시 혈압을 일정하게 유지하기 위해서는 vestibular sympathetic reflex 가 정상적으로 작동하여야 하는데, 자율 신경계의 조절 부전 (sympathoneural autonomic dysfunction)으로 이 기능이 저하되면 잔존 어지럼을 느끼게 된다고 제안하였다.

이렇듯 현재까지 여러 요인들이 잔존 어지럼의 원인으로 제시되어 있다. 이러한 연구 결과에 따르면 잔존 어지럼은 한 
가지 원인에 의해 발생하는 단일 질병이라기 보다는 여러 가 지 요인에 의해 유발되는 증후군으로 보인다(Fig. 1). 잔존 어 지럼의 발생 기전을 밝히기 위해서는 추가적인 연구가 필요 할 것이다.

\section{잔존 어지럼의 예측}

$\mathrm{BPPV}$ 는 비교적 양호한 예후를 보이지만, 환자는 극심한 어지럼을 경험하게 되어 $\mathrm{BPPV}$ 의 경과에 많은 궁금증을 가 지게 된다. ${ }^{30)}$ 따라서 잔존 어지럼을 포함한 $\mathrm{BPPV}$ 의 경과를 예측할 수 있다면 BPPV 환자 진료에 큰 도움을 줄 수 있을 것이다. cVEMP와 골다공증의 유무를 이용해서 잔존 어지럼 을 예측한 연구가 있었다.

$\mathrm{Oh}$ 등 $^{311}$ 은 65 명의 BPPV 환자를 잔존 어지럼 유무에 따라 분류하였다. 그후 나이, 성별, $\mathrm{BPPV}$ 가 발생한 세반고리관, $\mathrm{BPPV}$ 가 발생한 방향(좌우), $\mathrm{BPPV}$ 의 지속 기간, cVEMP 결 과(BPPV 완치 전 시행) 중 잔존 어지럼 발생에 유의한 영향 을 미치는 인자를 다중 회귀 분석을 통해 분석하였다. 그 결 과 cVEMP-modified interaural amplitude difference ratio 가 잔존 어지럼 발생과 유의하게 연관되어 있는 것으로 보고 하였고, cVEMP 결과를 이용하여 잔존 어지럼 발생을 예측 할 수 있을 것으로 제안하였다.

Suh 등 ${ }^{32}$ 은 62명의 BPPV 환자를 잔존 어지럼 유무에 따라 분류하였다. 그후 나이, 성별, $\mathrm{BPPV}$ 가 발생한 세반고리관, $\mathrm{BPPV}$ 가 발생한 방향(좌우), $\mathrm{BPPV}$ 의 지속 기간, 고혈압, 당 뇨병, 고지혈증, 골다공증의 유무 중 잔존 어지럼 발생에 유 의한 영향을 미치는 인자를 다중 회귀 분석을 통해 분석하 였다. 그 결과 골다공증이 있는 BPPV 환자에서 잔존 어지럼 이 유의하게 더 많이 발생함을 보였다. 이를 통해 BPPV 환자 가 골다공증이 있을 경우 잔존 어지럼 발생 가능성이 더 높 음을 제안하였다.

두 연구 모두 잔존 어지럼 발생의 기전이 이석 기관 장애일

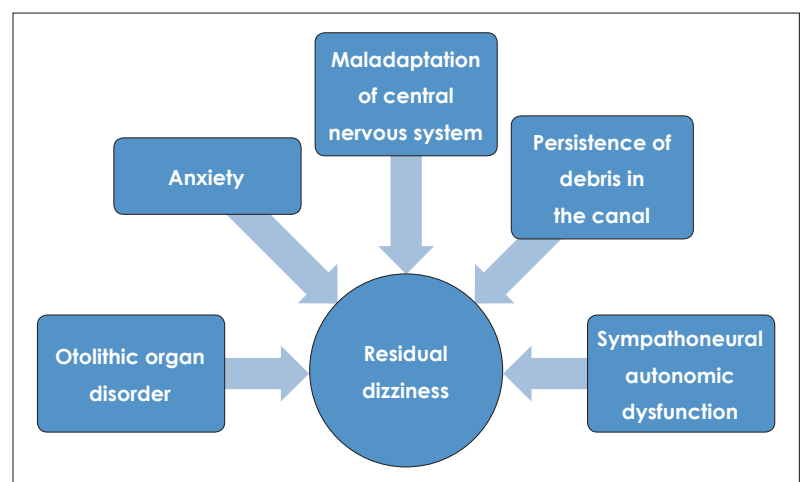

Fig. 1. Factors associated with residual dizziness.
것이라는 가설에서 출발하였다. Oh 등 ${ }^{31}$ 의 연구는 cVEMP 결과가 이석 기관 장애 여부를 반영할 것이라고 가정하였고, 이석 기관 장애가 있는 $\mathrm{BPPV}$ 환자에서 잔존 어지럼이 더 많 이 발생할 것으로 가정하고 이를 보여주었다. Suh 등 $^{32}$ 의 연 구 역시 골다공증이 있는 사람에서 BPPV가 더 잘 발생한다 는 사실을 바탕으로, 골다공증이 있으면 이석 기관 장애가 더 잘 발생할 것이라고 가정하였다. 그리고 이석 기관 장애가 잔존 어지럼을 유발할 것이라고 가정하고 이를 보여주었다. 앞으로 추가적인 연구가 이루어진다면 잔존 어지럼의 발생을 미리 예측할 수도 있을 것이다. 그리고 이런 연구 결과들은 잔존 어지럼과 이석 기관 장애 사이의 연관성을 뒷받침하는 또 다른 근거이다.

\section{잔존 어지럼에 있어 약물 치료의 효과}

잔존 어지럼의 치료법을 찾고자 현재까지 몇 가지 임상 시 험이 진행되었다. 전정 억제제(vestibular suppressant), 항불 안제(anxiolytics) 등이 사용되었고, 그 결과가 보고되어 있다. Acar 등 ${ }^{33}$ 은 trimetazidine, betahistine, ginkgo biloba extract 투여가 잔존 어지럼 완화에 도움을 주는지 임상 시험을 진행하였다. $\mathrm{BPPV}$ 진단 후 성공적인 이석정복술을 시행 받은 환자 100명을 네 그룹으로 나누고, 세 그룹은 각각 trimetazidine, betahistine, ginkgo biloba extract을 1주일간 복용하 고 한 그룹은 아무런 약도 복용하지 않았다. 이석정복술 시행 1, 3, 5일 후 Dizziness Handicap Inventory(DHI) 설문지를 작성하였다. 이석정복술 시행 3,5 일 후 DHI 점수는 그룹 간 에 유의한 차이가 없었다고 보고하여, trimetazidine, betahistine, ginkgo biloba extract 등의 약물 치료가 잔존 어지 럼에 효과가 없는 것으로 보고하였다.

Kim 등 ${ }^{34}$ 은 dimenhydrinate 투여가 잔존 어지럼 완화에 도움을 주는지 임상 시험을 진행하였다. $\mathrm{BPPV}$ 로 진단 후 성 공적인 이석정복술을 시행받은 138 명의 환자를 3 개의 군으로 나누었다: 대조군, dimenhydrinate 투여군, placebo 투여군. 1 주일 후 $\mathrm{DHI}$ 설문지를 작성하고, 잔존 어지럼이 있는지 물 었다. DHI 점수에서는 군 간에 차이가 없었으나, 잔존 어지럼 유무에서는 dimenhydrinate 투여군이 유의하게 우수한 결 과를 보여 잔존 어지럼 치료에 dimenhydrinate가 효과가 있 음을 제시하였다. 또한 DHI 설문지가 잔존 어지럼 평가에는 적절하지 않다고 주장하였다.

Jung 등은 항불안제가 잔존 어지럼 치료에 도움이 되는지 연구를 진행하였다. BPPV로 진단 후 성공적인 이석정복술을 시행받은 73 명의 환자를 대조군과 실험군으로 나눴다. 대조 군에는 약물을 투여하지 않았고, 실험군에는 2주간 etizol- 
$\mathrm{am}$ 을 투여하였다. 이석정복술 시행 전에 DHI 설문지를 시행 하고, 이석정복술 시행 2주 후에 DHI 설문지를 시행하였다. 두 그룹 모두에서 $\mathrm{DHI}$ 점수가 유의하게 감소하였는데, functional, emotional subscale score는 실험군에서 유의하게 더 감소하였다. 이를 바탕으로 저자들은 항불안제가 $\mathrm{BPPV}$ 후 증상 개선에 도움이 될 것이라고 제시하였다. 하지만 잔존 어 지럼이 아니라 불안감을 낮춘 것이라는 지적이 있었다.

\section{결 론}

잔존 어지럼은 아직까지 치료 방법은 물론 발생 기전도 명 확히 알려지지 않은 질환이다. 하지만 $\mathrm{BPPV}$ 환자의 약 절반 에서 발생할 정도로 매우 흔한 질환으로, 어지럼 환자를 진료 함에 있어 반드시 고려되어야 할 질환이다. 앞으로 많은 연구 가 이루어질 수 있는 분야로 어지럼을 연구하는 연구자들의 많은 관심이 필요하다.

\section{Acknowledgments}

None.

\section{Author Contribution}

Conceptualization: Munyoung Chang, Seog-Kyun Mun. Investigation: Munyoung Chang, Seog-Kyun Mun. Methodology: Munyoung Chang, Seog-Kyun Mun. Project administration: Munyoung Chang, Seog-Kyun Mun. Supervision: Munyoung Chang, SeogKyun Mun. Writing — original draft: Munyoung Chang. Writingreview \& editing: Seog-Kyun Mun.

\section{ORCIDs}

Seog-Kyun Mun

https://orcid.org/0000-0001-8624-2964

Munyoung Chang

https://orcid.org/0000-0003-0136-3893

\section{REFERENCES}

1) von Brevern M, Radtke A, Lezius F, Feldmann M, Ziese T, Lempert $\mathrm{T}$, et al. Epidemiology of benign paroxysmal positional vertigo: A population based study. J Neurol Neurosurg Psychiatry 2007;78(7): $710-5$.

2) Bhattacharyya N, Baugh RF, Orvidas L, Barrs D, Bronston LJ, Cass $\mathrm{S}$, et al. Clinical practice guideline: Benign paroxysmal positional vertigo. Otolaryngol Head Neck Surg 2008;139(5 Suppl 4): S47-81

3) Kim JS, Zee DS. Clinical practice. Benign paroxysmal positional vertigo. N Engl J Med 2014;370(12):1138-47.

4) Seok JI, Lee HM, Yoo JH, Lee DK. Residual dizziness after successful repositioning treatment in patients with benign paroxysmal positional vertigo. J Clin Neurol 2008;4(3):107-10.

5) Jung HJ, Koo JW, Kim CS, Kim JS, Song JJ. Anxiolytics reduce residual dizziness after successful canalith repositioning maneuvers in benign paroxysmal positional vertigo. Acta Otolaryngol 2012; 132(3):277-84

6) Teggi R, Quaglieri S, Gatti O, Benazzo M, Bussi M. Residual dizziness after successful repositioning maneuvers for idiopathic benign paroxysmal positional vertigo. ORL J Otorhinolaryngol Relat Spec 2013;75(2):74-81.
7) Kim HA, Lee H. Autonomic dysfunction as a possible cause of residual dizziness after successful treatment in benign paroxysmal positional vertigo. Clin Neurophysiol 2014;125(3):608-14.

8) Singh NK, Apeksha K. Efficacy of cervical and ocular vestibularevoked myogenic potentials in evaluation of benign paroxysmal positional vertigo of posterior semicircular canal. Eur Arch Otorhinolaryngol 2016;273(9):2523-32.

9) Faralli M, Lapenna R, Giommetti G, Pellegrino C, Ricci G. Residual dizziness after the first BPPV episode: Role of otolithic function and of a delayed diagnosis. Eur Arch Otorhinolaryngol 2016;273(10): 3157-65.

10) Oghalai JS, Manolidis S, Barth JL, Stewart MG, Jenkins HA. Unrecognized benign paroxysmal positional vertigo in elderly patients. Otolaryngol Head Neck Surg 2000;122(5):630-4.

11) Pritcher MR, Whitney SL, Marchetti GF, Furman JM. The influence of age and vestibular disorders on gaze stabilization: A pilot study. Otol Neurotol 2008;29(7):982-8.

12) Teggi R, Giordano L, Bondi S, Fabiano B, Bussi M. Residual dizziness after successful repositioning maneuvers for idiopathic benign paroxysmal positional vertigo in the elderly. Eur Arch Otorhinolaryngol 2011;268(4):507-11.

13) Brandt T. Phobic postural vertigo. Neurology 1996;46(6):1515-9.

14) Huppert D, Strupp M, Rettinger N, Hecht J, Brandt T. Phobic postural vertigo--a long-term follow-up ( 5 to 15 years) of 106 patients. J Neurol 2005;252(5):564-9.

15) Seo T, Shiraishi K, Kobayashi T, Mutsukazu K, Fujita T, Saito K, et al. Residual dizziness after successful treatment of idiopathic benign paroxysmal positional vertigo originates from persistent utricular dysfunction. Acta Otolaryngol 2017;137(11):1149-52.

16) von Brevern $M$, Schmidt T, Schönfeld U, Lempert T, Clarke AH. Utricular dysfunction in patients with benign paroxysmal positional vertigo. Otol Neurotol 2006;27(1):92-6.

17) Yetiser S, Ince D, Gul M. An analysis of vestibular evoked myogenic potentials in patients with benign paroxysmal positional vertigo. Ann Otol Rhinol Laryngol 2014;123(10):686-95.

18) Gacek RR. Pathology of benign paroxysmal positional vertigo revisited. Ann Otol Rhinol Laryngol 2003;112(7):574-82.

19) Hoseinabadi R, Pourbakht A, Yazdani N, Kouhi A, Kamali M. The effects of abnormality of cVEMP and oVEMP on rehabilitation outcomes in patients with idiopathic benign paroxysmal positional vertigo. Eur Arch Otorhinolaryngol 2016;273(3):643-8.

20) Korres S, Gkoritsa E, Giannakakou-Razelou D, Yiotakis I, Riga M, Nikolpoulos TP. Vestibular evoked myogenic potentials in patients with BPPV. Med Sci Monit 2011;17(1):CR42-7.

21) Welling DB, Parnes LS, O’Brien B, Bakaletz LO, Brackmann DE, Hinojosa R. Particulate matter in the posterior semicircular canal. Laryngoscope 1997;107(1):90-4.

22) Halmagyi GM, Curthoys IS. Clinical testing of otolith function. Ann N Y Acad Sci 1999;871:195-204.

23) Böhmer A, Rickenmann J. The subjective visual vertical as a clinical parameter of vestibular function in peripheral vestibular diseases. J Vestib Res 1995;5(1):35-45.

24) Gall RM, Ireland DJ, Robertson DD. Subjective visual vertical in patients with benign paroxysmal positional vertigo. J Otolaryngol 1999;28(3):162-5.

25) Colebatch JG, Halmagyi GM. Vestibular evoked potentials in human neck muscles before and after unilateral vestibular deafferentation. Neurology 1992;42(8):1635-6.

26) Faralli M, Ricci G, Ibba MC, Crognoletti M, Longari F, Frenguelli A. Dizziness in patients with recent episodes of benign paroxysmal positional vertigo: Real otolithic dysfunction or mental stress? J Otolaryngol Head Neck Surg 2009;38(3):375-80.

27) Inagaki T, Suzuki M, Otsuka K, Kitajima N, Furuya M, Ogawa Y, et al. Model experiments of BPPV using isolated utricle and posterior 
semicircular canal. Auris Nasus Larynx 2006;33(2):129-34.

28) Prokopakis EP, Lachanas VA, Christodoulou PN, Velegrakis GA. Dizziness after canalith repositioning procedure for benign paroxysmal positional vertigo. Auris Nasus Larynx 2007;34(3):435; author reply 437.

29) Di Girolamo S, Ottaviani F, Scarano E, Picciotti P, Di Nardo W. Postural control in horizontal benign paroxysmal positional vertigo. Eur Arch Otorhinolaryngol 2000;257(7):372-5.

30) Park MK, Lee DY, Kim YH. Risk factors for positional vertigo and the impact of vertigo on daily life: The Korean national health and nutrition examination survey. J Audiol Otol 2019;23(1):8-14.

31) Oh KH, Suh KD, Lee YH, Lee SY, Chang MY, Mun SK. Clinical utility of cervical vestibular-evoked myogenic potentials in predicting residual dizziness after benign paroxysmal positional vertigo. Clin Neurophysiol 2019;130(1):95-100.

32) Suh KD, Oh SR, Chae H, Lee SY, Chang M, Mun SK. Can osteopenia induce residual dizziness after treatment of benign paroxysmal positional vertigo? Otol Neurotol 2020;41(5):e603-6.

33) Acar B, Karasen RM, Buran Y. Efficacy of medical therapy in the prevention of residual dizziness after successful repositioning maneuvers for benign paroxysmal positional vertigo (BPPV). B-ENT 2015;11(2):117-21.

34) Kim MB, Lee HS, Ban JH. Vestibular suppressants after canalith repositioning in benign paroxysmal positional vertigo. Laryngoscope 2014;124(10):2400-3.

\section{정답 및 해설}

1. 답 (3)

해 설 흡연남성에게 제일 흔하게 발생하는 종양은 Warthin tumor이다.

2. 답 (1)

해 설 소아에서 midline에 가장 흔하게 발생하는 선천성 경부 종양은 갑상설관 낭종이다. 